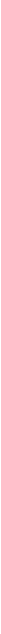

5
5
0
$\Sigma$
$\Sigma$

\title{
TOWARD A THEORY OF FUZZY SYSTEMS
}

by L. A. Zadeh

\author{
Prepared by \\ UNIVERSITY OF CALIFORNIA \\ Berkeley, Calif. \\ for
}

NATIONAL AERONAUTICS AND SPACE ADMINISTRATION - WASHINGTON, D. C. - SEPTEMBER 1969 
TOWARD A THEORY OF FUZZY SYSTEMS

By L. A. Zadeh

Distribution of this report is provided in the interest of information exchange. Responsibility for the contents resides in the author or organization that prepared it.

Issued by Originator as Report No. ERL-69-2

Prepared under Grant No. NGL-05-003-016 by ELECTRONICS RESEARCH LABORATORY UNIVERSITY OF CALIFORNIA

Berkeley, Calif.

for

\section{NATIONAL AERONAUTICS AND SPACE ADMINISTRATION}

For sale by the Clearinghouse for Federal Scientific and Technical Information Springfield, Virginia 22151 - CFSTI price $\$ 3.00$ 


\section{ABSTRACT}

Many of the systems encountered in the real world are too complex and/or too ill-defined to be susceptible of exact analysis. The concept of a fuzzy set, that is, a class which admits of intermediate grades of membership in it, opens the possibility of analyzing such systems both qualitatively and quantitatively by allowing the input and/or the output and/or the state of the system to range over fuzzy sets. In this paper, several basic concepts relating to the characterization of discrete-time fuzzy systems are introduced and input-output-state equations for such systems are developed. 
. 


\section{CONTENTS}

Page No.

INTRODUCTION

1

CHAPTERS

I. Elementary Properties of Fuzzy Sets 4

II. System, Aggregate and State 11

III. State Equations for Fuzzy Systems 13

IV. Fuzzy Systems and Fuzzy Algorithms 26

V. The Concept of Aggregate 30

$\begin{array}{ll}\text { BIBL IOGRAPHY } & 35\end{array}$ 


\section{INTRODUCTION}

Many of the advances in network theory and system theory during the past three decades are traceable to the influence and contributions of Ernst Guillemin, Norbert Wiener, Richard Bellman, Rudolph Kalman, and their students. In sum, we now possess an impressive armamentarium of techniques for the analysis and synthesis of linear and nonlinear systems of various types - techniques which are particularly effective in dealing with systems characterized by ordinary differential or difference equations of moderately high order such as are encountered in network theory, control theory and related fields.

What we still lack, and lack rather acutely, are methods for dealing with systems which are too complex or too ill-defined to admit of precise analysis. Such systems pervade life sciences, social sciences, philosophy, economics, psychology and many other "soft" fields. Furthermore, they are encountered in what are normally regarded as "nonsoft" fields when the complexity of a system rules out the possibility of analyzing it by conventional mathematical means, whether with or without the aid of computers. Many examples of such systems are found among large-scale traffic control systems, pattern recognition systems, machine translators, large-scale information processing systems, large-scale power distribution networks, neural networks and games such as chess, checkers, etc.

Perhaps the major reason for the ineffectiveness of classical mathematical techniques in dealing with systems of high order of complexity lies in their failure to come to grips with the issue of fuzziness, that is, with imprecision which stems not from randomness but from a lack of sharp transition from membership in a class to non-membership in it. It is this type of imprecision which arises when one speaks, for example, of the class of real numbers which are much larger than 10 , since the real numbers cannot be divided dichotomously into those that are much larger than 10 and those that are not. The same applies to classes such as "tall men," "good strategies for playing chess," "pairs of numbers which are approximately equal to one another," "systems which 
are approximately linear," etc. Actually, most of the classes encountered in the real world are of this fuzzy, imprecisely defined, kind. What sets such classes apart from classes which are well defined in the conventional mathematical sense is the fuzziness of their boundaries. In effect, in the case of a class with a fuzzy boundary, an object may have a grade of membership in it which lies somewhere between full membership and non-membership.

A class which admits of the possibility of partial membership in it is called a fuzzy set. ${ }^{1}$ In this sense, the class of tall men, for example, is a fuzzy set, as is the class of real numbers which are much larger than 10. We make a fuzzy statement or assertion when some of the words appearing in the statement or assertion in question are names for fuzzy sets. This is true, for example, of statements such as "John is tall," "x is approximately equal to 5," "y is much larger than 10 ," etc. In these statements, the sources of fuzziness are the underlined words, which, in effect, are labels for fuzzy sets. Why is fuzziness so relevant to complexity? Because no matter what the nature of a system is, when its complexity exceeds a certain threshold it becomes impractical or computationally infeasible to make precise assertions about it. For example, in the case of chess the size of the decision tree is so large that it is impossible, in general, to find a precise algorithmic solution to the following problem: Given the position of pieces on the board, determine an optimal next move. Similarly, in the case of a large-scale traffic control system, the complexity of the system precludes the possibility of precise evaluation of its performance. Thus, any significant assertion about the performance of such a system must necessarily be fuzzy in nature, with the degree of fuzziness increasing with the complexity of the system.

How can fuzziness be made a part of system theory? A tentative step in this direction was taken in recent papers ${ }^{2,3}$ in which the notions of a fuzzy system* and fuzzy algorithm were introduced. In what

\footnotetext{
* The maximin automata of Wee and Santos (Refs. 4 and 5) may be regarded as instances of fuzzy systems.
} 
follows, we sha11 proceed somewhat further in this direction, focusing our attention on the definition of a fuzzy system and its state. It should be emphasized, however, that the task of constructing a complete theory of fuzzy systems is one of very considerable magnitude, and that what we shall have to say about fuzzy systems in the sequel is merely a first step toward devising a conceptual framework for dealing with such systems in both qualitative and quantitative ways. 


\section{CHAPTER I}

\section{ELEMENTARY PROPERTIES OF FUZZY SETS}

The concept of a fuzzy system is intimately related to that of a fuzzy set. In order to make our discussion self-contained, it will be helpful to begin with a brief summary of some of the basic definitions pertaining to such sets.*

Definition of a fuzzy set. Let $X=\{x\}$ denote a space of points (objects), with $x$ denoting a generic element of $X$. Then a fuzzy set $A$ in $X$ is a set of ordered pairs

$$
A=\left\{\left(x, \mu_{A}(x)\right)\right\}, x \in X
$$

where $\mu_{A}(x)$ is termed the grade of membership of $x$ in $A$. Thus, if $\mu_{A}(x)$ takes values in a space $M$ - termed the membership space - then $A$ is essentially a function from $X$ to $M$. The function $\mu_{A}: X \rightarrow M$ which defines $A$ is called the membership function of $A$. When $M$ contains only two points 0 and 1 , $A$ is non-fuzzy and its membership function reduces to the conventional characteristic function of a non-fuzzy set.

Intuitively, a fuzzy set $A$ in $X$ is a class without sharply defined boundaries, that is, a class in which a point (object) $x$ may have a grade of membership intermediate between ful1 membership and non-membership. The important point to note is that such a fuzzy set can be defined precisely by associating with each $x$ its grade of membership in $A$. In what follows, we sha11 assume for simplicity that $M$ is the interva1 $[0,1]$, with the grades 0 and 1 representing, respectively, non-membership and

\footnotetext{
* More detailed discussions of fuzzy sets and their properties may be found in the references listed in the Bibliography.
} 
full membership in a fuzzy set. (More generally, $M$ can be a partially ordered set or, more particularly, a lattice. ${ }^{6}$ ) Thus, our basic assumption.will be that a fuzzy set $A$ in $X$, even though lacking in sharply defined boundarles, can be precisely characterlzed by a membership function which associates with each $x$ in $X a$ number in the interval $[0,1]$ representing the grade of membership of $x$ in $A$.

Example. Let $A=\{x \mid x>1\}$ (i.e., $A$ is the fuzzy set of real numbers which are much larger than 1). Such a set may be defined subjectively by a membership function such as:

$$
\begin{aligned}
& \mu_{A}(x)=0 \quad \text { for } x \leq 1 \\
& =\left(1+(x-1)^{-2}\right)^{-1} \quad \text { for } \quad x>1
\end{aligned}
$$

It is important to note that in the case of a fuzzy set it is not meaningful to speak of an object as belonging or not belonging to that set, except for objects whose grade of membership in the set is unity or zero. Thus, if $A$ is the fuzzy set of tall men, then the statement "John is tall" should not be interpreted as meaning that John belongs to $A$. Rather, such a statement should be interpreted as an association of John with fuzzy set A - an association which will be denoted by John $\underset{\sim}{\in}$ to distinguish it from an assertion of belonging in the usual non-fuzzy sense, that is, John $\in A$, which is meaningful only when $A$ is non-fuzzy. *

Containment. Let $A$ and $B$ be fuzzy sets in $X$. Then $A$ is contained in $B$ (or $A$ is a subset of $B$ ) written as $A \subset B$, if and only if $\mu_{A}(x) \leq \mu_{B}(x)$

\footnotetext{
* Here and elsewhere in this paper we shall employ the convention of underscoring a symbol with a wavy bar to represent a fuzzified version of the meaning of that symbol. For example, $x \equiv y$ will denote a fuzzy equality of $x$ and $y ; x \rightarrow y$ will denote fuzzy implication, etc.
} 
for all $x$ in $x$. (In the sequel, to simplify the notation we shall omit $x$ when an equality or inequality holds for all values of $x$ in $x$. )

Example. If $\mu_{A}=\mu_{B}^{2}$, then $A \subset B$.

Equality. Two fuzzy sets are equal, written as $A=B$, if and only if $\mu_{A}=\mu_{B} \cdot$

Complementation. A fuzzy set $A^{\prime}$ is the complement of a fuzzy set $A$ if and only if $\mu_{A^{\prime}}=1-\mu_{A^{\prime}}$.

Example. The fuzzy sets $A=\{x \mid x>1\}$ and $A^{\prime}=\{x \mid x$ not $>1\}$ are complements of one another.

Union. The union of $A$ and $B$ is denoted by $A \cup B$ and is defined as the smallest fuzzy set containing both $A$ and $B$. The membership function of $A \cup B$ is given by $\mu_{A \cup B}=\operatorname{Max}\left[\mu_{A}, \mu_{B}\right]$. Thus, if at a point $x, \mu_{A}(x)=0.9$, say, and $\mu_{B}(x)=0.4$, then at that point $\mu_{A \cup B}(x)=0.9$.

As in the case of non-fuzzy sets, the notion of the union is closely related to that of the connective "or". Thus, if A is a class of tall men, B is a class of fat men and "John is tall" or "John is fat," then John is associated with the union of A and B. More generally, expressed in symbols, we have

$$
\mathrm{x} \underset{\sim}{\in} \mathrm{A} \quad \text { or } \quad \mathrm{x} \underset{\sim}{\in} \mathrm{B} \rightarrow \mathrm{x} \underset{\sim}{\in} \mathrm{A} \cup \mathrm{B}
$$

Intersection. The intersection of $A$ and $B$ is denoted by $A \cap B$ and is defined as the largest fuzzy set contained in both $A$ and $B$. The membership function of $A \cap B$ is given by $\mu_{A \cap B}=\operatorname{Min}\left[\mu_{A}, \mu_{B}\right]$. It is easy to verify that $A \cap B=\left(A^{\prime} \cap B^{\prime}\right)^{\prime}$. The relation between the connective "and" and $\cap$ is expressed by 
$\mathbf{x} \in \mathbf{A} \quad$ and $\quad \mathrm{x} \underset{\sim}{\in} \mathrm{B} \rightarrow \mathrm{x} \underset{\sim}{\in} \mathrm{A} \cap \mathrm{B}$

Algebraic product. The algebraic product of $A$ and $B$ is denoted by $A B$ and is defined by $\mu_{A B}=\mu_{A} \mu_{B}$.

Algebraic sum. The algebraic sum of $A$ and $B$ is denoted by $A \oplus B$ and is defined by $\mu_{A \oplus B}=\mu_{A}+\mu_{B}-\mu_{A B}$. It is trivial to verify that $A \oplus B=$ $\left(A^{\prime} B^{\prime}\right)^{\prime}$.

Relation. A fuzzy relation, $R$, in the product space $X \times Y=\{(x, y)\}$, $\mathrm{X} \in \mathrm{X}, \mathrm{y} \in \mathrm{Y}$, is a fuzzy set. in $\mathrm{X} \times \mathrm{Y}$ characterized by a membership function $\mu_{R}$ which associates with each ordered pair $(x, y)$ a grade of membership $\mu_{R}(x, y)$ in $R$. More generally, an $n$-ary fuzzy relation in a product space $\mathrm{X}=\mathrm{X}^{1} \times \mathrm{X}^{2} \times \ldots \times \mathrm{X}^{\mathrm{n}}$ is a fuzzy set in $\mathrm{X}$ characterized by an n-variate membership function $\mu_{R}\left(x_{1}, \ldots, x_{n}\right), x_{i} \in x^{i}, i=1, \ldots, n$.

Example. Let $X=Y \times R$, where $R$ is the real line $(-\infty, \infty)$. Then $x>y$ is a fuzzy relation in $R^{2}$. A subjective expression for $\mu_{R}$ in this case might be: $\mu_{R}(x, y)=0$ for $x \leq y ; \mu_{R}(x, y)=\left(1+(x-y)^{-2}\right)^{-1}$ for $x>y$.

Composition of relations. If $R_{1}$ and $R_{2}$ are two fuzzy relations in $x^{2}$, then by the composition of $R_{1}$ and $R_{2}$ is meant a fuzzy relation in $x^{2}$ which is denoted by $R_{1} \circ R_{2}$ and is defined by

$$
\mu_{R_{1} \circ R_{2}}(x, y)=\operatorname{Sup}_{v} \operatorname{Min}\left(R_{1}(x, v), R_{2}(v, y)\right)
$$

where the supremum is taken over all $v$ in $x$.

Fuzzy sets induced by mappings. Let $f: X \rightarrow Y$ be a mapping from $X$ to $Y$, with the image of $x$ under $f$ denoted by $y=f(x)$. Let $A$ be a fuzzy set in $x$. Then, the mapping $f$ induces a fuzzy set $B$ in $Y$ whose membership function 
is given by

$$
\mu_{B}(y)=\operatorname{Sup}_{x \in f^{-1}(y)} \mu_{A}(x)
$$

where $f^{-1}(y)$ denotes the set of points in $x$ which are mapped by $f$ into $y$.

Shadow of a fuzzy set. Let $A$ be a fuzzy set in $X \times Y$, and let $f$ denote the mapping which takes $(x, y)$ into $x$. The fuzzy set in $X$ which is induced by this mapping is called the shadow (projection) of $A$ on $X$ and is denoted by $S_{X}(A)$. In consequence of $(1.6)$, the membership function of $S_{X}(A)$ is given by

$$
\mu_{S}(A)=\operatorname{Sup}_{y} \mu_{A}(x, y)
$$

where $\mu_{A}(x, y)$ is the membership function of $A$.

Conditioned fuzzy sets. A fuzzy set $B(X)$ in $Y$ will be said to be conditioned on $x$ if its membership function depends on $x$ as a parameter. To place this dependence in evidence, we shall denote the membership function of $B(x)$ as $\mu_{B}(y \mid x)$ or - when $B$ can be omitted with no risk of confusion - as $\mu(y \mid x)$.

Now suppose that the parameter $x$ ranges over a space $X$. Then, the function $\mu_{B}(y \mid x)$ defines a mapping from $X$ to the space of fuzzy sets defined on $Y$. Through this mapping, a fuzzy set $A$ in $X$ induces a fuzzy set $B$ in $Y$ which is defined by

$$
\mu_{B}(y)=\sup _{x \in X} \operatorname{Min}\left(\mu_{A}(x), \mu_{B}(y \mid x)\right)
$$

where $\mu_{A}$ and $\mu_{B}$ denote the membership functions of $A$ and $B$, respectively. 
In effect, (1.8) is a special case of the composition of relations (1.5).

The notion of a conditioned fuzzy set bears some resemblance to the notion of a conditional probability distribution. Thus, (1.8) is the counterpart of the familiar identity

$$
p_{B}(y)=\int_{x} \quad p_{B}(y \mid x) p_{A}(x) d x
$$

where for simplicity $x$ and $y$ are assumed to be real-valued, $p_{A}(x)$ denotes the probability density of $x, p_{B}(y \mid x)$ denotes the conditional probability density of $y$ given $x$ and $p_{B}(y)$ denotes the probability density of $y . *$ It is worthy of note that, in this as well as many other instances involving fuzziness on the one hand and probability on the other, the corresponding formulae differ from one another in that to the operations of summation and integration involving probabilities corresponds the operation of taking the supremum (or maximum) of membership functions, and to the operation of multiplication of probabilities corresponds the operation of taking the infimum (or minimum) of membership functions. To make this correspondence more evident, it is convenient to use the symbols $V$ and $\wedge$ for the supremum and infimum, respectively. Then, (1.9) becomes

$$
\mu_{B}(y)=\underset{x}{V}\left(\mu_{A}(x) \wedge \mu_{B}(y) x\right)
$$

Similarly, (1.7) becomes

$$
\mu_{B}(y)=\underset{x \in f^{-1}(y)}{V}{ }_{A}(x)
$$

* To simplify the notation, we use the same symbol to denote a random variable and a generic value of that variable. 
for which its probabilistic counterpart reads

$$
p_{B}(y)=\sum_{x \in f^{-1}(y)} p_{A}(x)
$$

where $x$ and $y$ are assumed to range over finite sets and $p_{A}(x)$ and $p_{B}(y)$ denote probabilities rather than probability densities as in (1.9).

This concludes our brief summary of some of the basic concepts relating to fuzzy sets. In what follows, we shall employ these concepts in defining a fuzzy system and explore some of the elementary properties of such systems. 


\section{CHAPTER II}

SYSTEM, AGGREGATE AND STATE

For simplicity, we shall restrict our attention to timeinvarlant discrete-time systems in which $t$, time, ranges over integers, and the input and output at time $t$ are real-valued.

In the theory of non-fuzzy discrete-time systems, it is customary to introduce the notion of state at the very outset by defining a system $a$ through its state equations:

$$
\begin{aligned}
x_{t+1} & =f\left(x_{t}, u_{t}\right) \quad t=\ldots,-1,0,1, \ldots \\
y_{t} & =g\left(x_{t}, u_{t}\right)
\end{aligned}
$$

where $u_{t}$ denotes the input at time $t, y_{t}$ is the output at time $t$ and $x_{t}$ is the state at time $t$, with the ranges of $u_{t}, y_{t}$ and $x_{t}$ denoted by $U$, $Y$ and $X$, respectively. In this way, $Q$ is characterized by two mappings, $f: X \times U \rightarrow \sum$ and $g: X \times U \rightarrow Y$. The space $X$ is called the state space of $Q$, and $a$ point $\alpha$ in $X$ is called a state of $a$.

Let $\mathbf{u}$ denote an input sequence starting at, say, $t=0$. Thus, $\mathbf{u}=u_{0} u_{1} \ldots u$ where $u_{t} \in U, t=0,1, \ldots, \ell, \ell=$ non-negative integer. The set of all sequences whose elements are drawn from $U$ will be denoted by $\mathrm{U}^{*}$.

Now, to each state $\alpha$ in $X$ and each input sequence $u=u_{0} u_{1} \ldots$ $\mathbf{u}_{\ell}$ in $\mathrm{U}^{*}$ will correspond an output sequence $\mathrm{y}=\mathrm{y}_{0} \mathrm{y}_{1} \cdots \mathrm{y}_{\ell}$ in $\mathrm{Y}^{*}$. The pair of sequences $(u, y)$ is called an input-output pair of length $\ell+1$. The totality of input-output pairs, $(u, y)$, of varying lengths which correspond to a particular state $\alpha$ in $X$ will be referred to as an aggregate of input- 
output pairs, or simply an aggregate, $Q(\alpha)$, with $\alpha$ playing the role of a label for this aggregate. The union

$$
a=\underset{\alpha \in \mathrm{X}}{\mathrm{v}} a(\alpha)
$$

represents the totality of input-output pairs which correspond to all the states of $a$. It is this totality of input-output pairs that we sha11 equate with $a$.

The fact that a state is merely a label for an aggregate suggests that the concept of an aggregate be accorded a central place among the basic concepts of system theory. This is done implicitly in [8] and [9], and explicitly in [10]. The point of departure in the theory developed in [8] is the definition of a system as a collection of input-output pairs. An aggregate, then, may be defined as a subset of input-output pairs which satisfy certain consistency conditions, with a state playing the role of a name for an aggregate.

In what follows, we shall first generalize to fuzzy systems the conventional approach in which a system is described through its state equations. Then we shall indicate a connection between the notion of a fuzzy algortthm and a fuzzy system. Finally, we shall present in a summary form some of the basic definitions relating to the notion of an aggregate and briefly touch upon their generalization to fuzzy systems. 


\section{STATE EQUATIONS FOR FUZZY SYSTEMS}

Let $u_{t}, y_{t}$ and $x_{t}$ denote, respectively, the input, output and state of a system $a$ at time $t$. Such a system is said to be deterministic if it is characterized by state equations of the form

$$
\begin{aligned}
x_{t+1}=f\left(x_{t}, u_{t}\right) & t=-1,0,1,2, \ldots,
\end{aligned}
$$

in which $f$ and $g$ are mappings from $X \times U$ to $X$ and $Y$, respectively.

$Q$ is said to be non-deterministic if $x_{t+1}$ and/or $y_{t}$ are not uniquely determined by $x_{t}$ and $u_{t}$. Let $x^{t+1}\left(x_{t}, u_{t}\right)$ and $Y^{t}\left(x_{t}, u_{t}\right)$ or $x^{t+1}$ and $\mathrm{Y}^{\mathrm{t}}$, for short, denote, respectively, the sets of possible values of $x_{t+1}$ and $y_{t}$, given $x_{t}$ and $u_{t}$. Then (3.1) and (3.2) can be replaced by equations of the form

$$
\begin{aligned}
x^{t+1} & =F\left(x_{t}, u_{t}\right) \\
y^{t} & =G\left(x_{t}, u_{t}\right)
\end{aligned}
$$

where $F$ and $G$ are mappings from $X \times U$ into the space of subsets of $\Sigma$ and $Y$, respectively. Thus, a non-deterministic system is characterized by equations of the form (3.3) and (3.4), in which $\mathrm{X}^{\mathrm{t}+1}$ and $\mathrm{Y}^{t}$ are subsets of $\mathrm{X}$ and $Y$, respectively.

The next step in the direction of further generalization is to assume that $X^{t+1}$ and $Y^{t}$ are fuzzy rather than non-fuzzy sets in $X$ and 
$Y$, respectively. In this case, we shall say that $Q_{\text {is a fuzzy discrete - }}$ time system. Clearly, such a system reduces to a non-deterministic system when $\mathrm{x}^{\mathrm{t}+1}$ and $\mathrm{Y}^{\mathrm{t}}$ are non-fuzzy sets. In turn, a non-deterministic system reduces to a deterministic system when $\mathrm{X}^{\mathrm{t}+1}$ and $\mathrm{Y}^{\mathrm{t}}$ are single points (singletons) in their respective spaces.

Let $\mu_{X}\left(x_{t+1} \mid x_{t}, u_{t}\right)$ and $\mu_{Y}\left(y_{t} \mid x_{t}, u_{t}\right)$ denote the membership functions of $x^{t+1}$ and $y^{t}$, respectively, given $x_{t}$ and $u_{t}$. Then we can say that $Q$ is characterized by the two membership functions $\mu_{x}\left(x_{t+1}\right.$ $\left.x_{t}, u_{t}\right)$ and $\mu_{Y}\left(y_{t} \mid x_{t}, u_{t}\right)$, which define conditioned fuzzy sets in $X$ and $Y$, respectively, involving $x_{t}$ and $u_{t}$ as parameters.

To illustrate, suppose that $x=R^{3}$. Then $Q$ is a fuzzy system if its characterization contains statements such as: "If an input $u_{t}=5$ is applied to $Q$ in state $x_{t}=(3,5,1)$ at time $t$, then the state of $A$ at time $t=1$ will be in the vicinity of the point $(7,3,5) . "$ Here the set of points in $X$ which lie in the vicinity of a given point $\alpha$ is a fuzzy set in $X$. Such a set may be characterized by a membership function such as

$$
\mu(x)=\exp -\frac{1}{k}\|x-\alpha\|
$$

where $\mathrm{x}$ is a point in $\mathrm{x},\|\mathrm{x}-\alpha\|$ denotes a norm of the vector $\mathrm{x}-\alpha$, and $\mathrm{k}$ is a positive constant.

By analogy with non-fuzzy systems, a fuzzy system $Q$ will be said to be memoryless if the fuzzy set $\mathrm{Y}^{\mathrm{t}}$ is independent of $\mathrm{x}_{\mathrm{t}}$, that is, if its membership function is of the form $\mu_{Y}\left(y_{t} \mid u_{t}\right)$. Just as a nonfuzzy memoryless system is characterized by a graph $y_{t}=g\left(u_{t}\right), u_{t} \in U$, so a fuzzy memoryless system is characterized by a fuzzy graph which is a family of fuzzy sets $\left\{\mathrm{Y}^{t}\left(\mathrm{u}_{\mathrm{t}}\right), \mathrm{u}_{\mathrm{t}} \in \mathrm{U}\right\}$. 
In the case of a memoryless system, to each point $u_{t}$ in $U$ corresponds a fuzzy set $Y^{t}\left(u_{t}\right)$, or $Y^{t}$ for short, in $Y$. Thus, we can write

$$
Y^{t}=G\left(u_{t}\right) \quad t=\ldots,-1,0,1,2, \ldots,
$$

where $G$ is a function from $R^{1}$ to the space of fuzzy sets in $Y$. Now in consequence of (1.8), this implies that if $U^{t}$ is a fuzzy set in $U$ characterized by a membership function $\mu_{U}\left(u_{t}\right)$, then to $U^{t}$ will correspond the fuzzy set $\mathrm{Y}^{\mathrm{t}}$ defined by the membership function

$$
\mu_{Y}\left(y_{t}\right)=\underset{u_{t}}{v}\left(u_{U}\left(u_{t}\right) \wedge \mu_{Y}\left(y_{t} \mid u_{t}\right)\right)
$$

where $V$ and $\wedge$ denote the supremum and minimum, respectively. Thus, (3.7) establishes a relation between $U^{t}$ and $Y^{t}$ which can be expressed as

$$
\mathrm{Y}^{\mathrm{t}}=\mathrm{G}_{\mathrm{o}}\left(\mathrm{U}^{\mathrm{t}}\right) \quad \mathrm{t}=\ldots,-1,0,1,2, \ldots,
$$

where $G_{0}$ is a function from the space of fuzzy sets in $U$ to the space of fuzzy sets in $Y$.

The important point to be noted here is that equation $(3.6)$, which expresses $\mathrm{Y}^{t}$ as a function of $u_{t}$, induces equation (3.8), which expresses $\mathrm{Y}^{\mathrm{t}}$ as a function of $\mathrm{U}^{\mathrm{t}}$. As should be expected, (3.8) reduces to (3.6) when $U^{t}$ is taken to be the singleton $\left\{u_{t}\right\}$.

Intuitively, (3.6) and (3.8) may be interpreted as follows. If $a$ is a fuzzy memoryless system, then to every non-fuzzy input $u_{t}$ at time $t$ corresponds a unique fuzzy output which is represented by a 
conditioned fuzzy set $Y^{t}$ in $Y$. The membership function of this fuzzy set is given by $\mu_{Y}\left(y_{t} \mid u_{t}\right)$.

If the input to $Q$ is fuzzy, i.e., is a fuzzy set $U^{t}$ in $U$, then the corresponding fuzzy output $\mathrm{Y}^{\mathrm{t}}$ is given uniquely by (3.8). The membership function for $\mathrm{Y}^{\mathrm{t}}$ is expressed by (3.7).

As a very simple example, suppose that $U$ and $Y$ are finite sets: $U=\{1,2,3\}$ and $Y=\{1,2,3\}$. Furthermore, suppose that if the input $u_{t}$ is 1 , then the output is a fuzzy set described verbally as "y is approximately equal to $1 . "$ Similarly, if $u_{t}=2$, then $y_{t}$ is approximately equal to 2 , and if $u_{t}=3$, then $y_{t}$ is approximately equal to 3 . More concretely, we assume that $\mu_{Y}\left(y_{t} \mid u_{t}\right)$ is defined by the table:

$$
\begin{array}{lllll}
\mu_{Y}(1 \mid 1)=1 ; & \mu_{Y}(2 \mid 1)=0.3 ; & \mu_{Y}(3 \mid 1)=0.1 ; \\
\mu_{Y}(1 \mid 2)=0.2 ; & \mu_{T}(2 \mid 2)=1 ; & \mu_{Y}(3 \mid 2)=0.2 ; \\
\mu_{Y}(1 \mid 3)=0.1 ; & \mu_{Y}(2 \mid 3)=0.2 ; & \mu_{Y}(3 \mid 3)=1 ;
\end{array}
$$

Now assume that the input is a fuzzy set described verbally as " $u_{t}$ is close to $1, "$ and characterized by the membership function

$$
\mu_{U}(1)=1 ; \quad \mu_{U}(2)=0.2 ; \quad \mu_{U}(3)=0.1 ;
$$

Then, by using (3.7) the response to this fuzzy input is found to be a fuzzy set defined by the membership function

$$
\mu_{Y}(1)=1 ; \mu_{Y}(2)=0.3 ; \mu_{Y}(3)=0.2 ;
$$


It is convenient to regard (3.8) as a mapping from names of fuzzy sets in $U$ to names of fuzzy sets in $Y$. In many cases of practical interest such a mapping can be adequately characterized by a finite, and perhaps even fairly small, number of points (ordered paris $(U, Y)$ ) on the graph of $G_{0}$. For example, $G_{0}$ might be characterized approximately by a table such as shown below. (For simplicity we suppress the subscript $t$ in $u_{t}$ and $\left.y_{t} \cdot\right)$

\begin{tabular}{|c|c|}
\hline$u^{t}$ & $\mathrm{Y}^{\mathrm{t}}$ \\
\hline 1 & 1 \\
\hline 1.1 & 1.3 \\
\hline 1.2 & 1.6 \\
\hline 1.3 & 2 \\
\hline 1.4 & 2.5 \\
\hline 1.5 & 2.9 \\
\hline 1.6 & 2.5 \\
\hline 1.7 & 2.1 \\
\hline 1.8 & 1.8 \\
\hline 1.9 & 1.6 \\
\hline 2 & 1.5 \\
\hline 2.1 & 1.5 \\
\hline$:$ & $:$ \\
\hline 3 & 1.5 \\
\hline
\end{tabular}

where $x, x \in R$, is the name for the fuzzy set of real numbers which are approximately equal to $x$. Such a set may be characterized quantitatively by a membership function. In many practical situations a very approximate description of this membership function would be sufficient. In this way, equation (3.8) can serve the purpose of an approximate characterization of a fuzzy memoryless system. 
Turning to non-memoryless fuzzy systems, consider a system $Q$ which is characterized by state equations of the form

$$
\begin{aligned}
x^{t+1} & =F\left(x_{t}, u_{t}\right) \\
Y^{t} & =G\left(x_{t}, u_{t}\right)
\end{aligned}
$$

where $F$ is a function from the product space $X \times U$ to the space of fuzzy sets in $X, G$ is a function from $X \times U$ to the space of fuzzy sets in $Y, X^{t+1}$ denotes a fuzzy set in $X$ which is conditioned on $x_{t}$ and $u_{t}$, and $Y^{t}$ denotes a fuzzy set in $Y$ which, like $X^{t+1}$, is conditioned on $x_{t}$ and $u_{t} \cdot X^{t+1}$ and $Y^{t}$ represent, respectively, the fuzzy state and output of $Q$ at time $t$ and are defined by the membership functions $\mu_{X}\left(x_{t+1} \mid x_{t}, u_{t}\right)$ and $\mu_{Y}\left(y_{t} \mid x_{t}, u_{t}\right)$.

Equations (3.9) and (3.10) relate the fuzzy state at time $t+1$ and the fuzzy output at time $t$ to the non-fuzzy state and non-fuzzy input at time $t$. As in the case of a memoryless system, we can deduce from these equations - by repeated application of (1.8) - the state equations for $Q$ for the case where the state at time $t$ or the input at time or both are fuzzy.

Specifically, let us assume that the state at time $t$ is a fuzzy set characterized by a membership function $\mu_{X}\left(x_{t}\right)$. Then, by applying (1.8), we deduce from (3.9) and (3.10).

$$
\begin{aligned}
\mu_{X}\left(x_{t+1}\right)= & \underset{x_{t}}{v}\left(\mu_{X}\left(x_{t}\right) \wedge \mu_{X}\left(x_{t+1} \mid x_{t}, u_{t}\right)\right) \\
\mu_{Y}\left(y_{t}\right)= & v\left(\mu_{X}\left(x_{t}\right) \wedge \mu_{Y}\left(y_{t} \mid x_{t}, u_{t}\right)\right) \\
& x_{t}
\end{aligned}
$$


which in symbolic form may be expressed as *

$$
\begin{aligned}
x^{t+1} & =F_{0}\left(x^{t}, u_{t}\right) \\
Y^{t} & =G_{0}\left(x^{t}, u_{t}\right)
\end{aligned}
$$

In what follows, to simplify the appearance of equations such as (3.11) and (3.12), we shall omit the subscripts $X$ and $Y$ in membership functions.

By $n$-fold iteration of (3.13) and (3.14), we can obtain expressions for $x^{t+n+1}$ and $x^{t+n}, n=1,2,3, \ldots$, in terms of $x^{t}$ and $u_{t}, \ldots$, $u_{t+n}$. For example, for $n=1$, we have

$$
\begin{aligned}
& x^{t+2}=F_{0}\left(F_{0}\left(X^{t}, u_{t}\right), u_{t+1}\right) \\
& Y^{t+1}=G_{0}\left(F_{0}\left(X^{t}, u_{t}\right), u_{t+1}\right)
\end{aligned}
$$

or more compactly

$$
\begin{aligned}
& x^{t+2}=F_{1}\left(x^{t}, u_{t}, u_{t+1}\right) \\
& Y^{t+1}=G_{1}\left(x^{t}, u_{t}, u_{t+1}\right)
\end{aligned}
$$

To express (3.17) and (3.18) in terms of membership functions, we note that on replacing $t$ with $t+1$ in (3.11) and (3.12), we obtain

\footnotetext{
* Note that $\mu_{X}\left(x_{t+1}\right)$ and $\mu_{X}\left(x_{t}\right)$ represent different membership functions. Strictly speaking, we should write them as $\mu_{x}{ }_{t+1}\left(x_{t+1}\right)$ and $\mu_{x}{ }\left(x_{t}\right)$, respectively.
} 


$$
\begin{aligned}
& \mu\left(x_{t+2}\right)=\operatorname{x}_{t+1}\left(\mu\left(x_{t+1}\right) \wedge \mu\left(x_{t+2} \mid x_{t+1}, u_{t+1}\right)\right) \\
& \mu\left(y_{t+1}\right)=x_{t+1}\left(\mu\left(x_{t+1}\right) \wedge \mu\left(y_{t+1} \mid x_{t+1}, u_{t+1}\right)\right)
\end{aligned}
$$

Then, on substituting $\mu\left(x_{t+1}\right)$ from (3.11) into (3.19) and (3.20), we get

$$
\mu\left(x_{t+2}\right)=\operatorname{x}_{t+1} v\left(\underset{x_{t}}{v}\left(\mu\left(x_{t}\right) \wedge \mu\left(x_{t+1} \mid x_{t}, u_{t}\right)\right) \wedge \mu\left(x_{t+2} \mid x_{t+1}, u_{t+1}\right)\right)
$$

and

$$
\mu\left(y_{t+1}\right)=v_{t+1}^{v}\left(x_{t}\left(\mu\left(x_{t}\right) \wedge \mu\left(x_{t+1} \mid x_{t}, u_{t}\right)\right) \wedge \mu\left(y_{t+1} \mid x_{t+1}, u_{t+1}\right)\right)
$$

which by virtue of the distributivity of $\vee$ and $\Lambda$ may be expressed as

$$
\mu\left(x_{t+2}\right)=\underset{x_{t+1}}{v} \underset{x_{t}}{\vee}\left(\mu\left(x_{t}\right) \wedge \mu\left(x_{t+1} \mid x_{t}, u_{t}\right) \wedge \mu\left(x_{t+2} \mid x_{t+1}, u_{t+1}\right)\right)
$$

$$
\mu\left(y_{t+1}\right)=\underset{x_{t+1}}{v} x_{t}^{V}\left(\mu\left(x_{t}\right) \wedge \mu\left(x_{t+1} \mid x_{t}, u_{t}\right) \wedge \mu\left(y_{t+1} \mid x_{t+1}, u_{t+1}\right)\right)
$$

and likewise for larger values of $\mathrm{n}$. It should be noted that these relations are fuzzy counterparts of the corresponding expressions for stochastic systems, ${ }^{11}$ with $\wedge$ and $\vee$ replacing product and sum, respectively, and membership functions replacing probability functions (see (3.6) et seq.).

In the above analysis, we have assumed that the successive inputs $u_{t}, \ldots, u_{t+n}$ are non-fuzzy. On this basis, we can obtain expressions 
for $x^{t+1}, \ldots, x^{t+n+1}$ and $y^{t}, \ldots, y^{t+n}$ in terms of $x^{t}$ and $u_{t}, \ldots, u_{t+n}$. It is natural to raise the question of what are the corresponding expressions for $x^{t+1}, \ldots, x^{t+n+1}$ and $Y^{t}, \ldots, y^{t+n}$ when the successive inputs are fuzzy.

First, let us focus our attention on the state equations (3.3) and (3.4), in which $F$ and $G$ are functions from $X \times U$ to fuzzy sets in $X$ and $Y$, respectively. Suppose that both the input at time $t$ and the state at time $t$ are fuzzy. What would be the expressions for the membership functions of $\mathrm{x}^{\mathrm{t}+1}$ and $\mathrm{y}^{\mathrm{t}}$ in this simple case?

Let $\mu\left(x_{t}, u_{t}\right)$ denote the membership function of the fuzzy set whose elements are ordered pairs $\left(x_{t}, u_{t}\right) \cdot{ }^{*}$ Then, using (1.8) we can express the membership functions of $x^{t+1}$ and $\mathrm{Y}^{\mathrm{t}}$ as follows:

$$
\begin{aligned}
\mu\left(x_{t+1}\right)= & v \underset{x_{t}}{v} u_{t}\left(\mu\left(x_{t}, u_{t}\right) \wedge \mu\left(x_{t+1} \mid x_{t}, u_{t}\right)\right) \\
\mu\left(y_{t}\right)= & v \underset{x_{t}}{v} u_{t}\left(\mu\left(x_{t}, u_{t}\right) \wedge \mu\left(y_{t} \mid x_{t}, u_{t}\right)\right)
\end{aligned}
$$

These formulae assume a simpler form when $\mu\left(x_{t}, u_{t}\right)$ can be expressed as

$$
\mu\left(x_{t}, u_{t}\right)=\mu\left(x_{t}\right) \wedge \mu\left(u_{t}\right)
$$

where $\mu\left(x_{t}\right)$ and $\mu\left(u_{t}\right)$ denote, respectively, the membership function of the fuzzy state and the fuzzy input at time $t$. In this case, we shall say that the fuzzy sets $x^{t}$ and $U^{t}$ are non-interacting. Essentially, the

* The probabllistic counterpart of this membership function is the joint probability of $x$ and $u$. 
notion of non-intersection of fuzzy sets corresponds to the notion of independence of random variables.

The assumption that $\mathrm{X}^{t}$ and $\mathrm{U}^{t}$ are non-interacting fuzzy sets is a reasonable one to make in many cases of practical interest. Under this assumption, $(3.25)$ and $(3.26)$ reduce to

$$
\begin{aligned}
\mu\left(x_{t+1}\right)= & v \quad v\left(\mu\left(x_{t}\right) \wedge \mu\left(u_{t}\right) \wedge \mu\left(x_{t+1} \mid x_{t}, u_{t}\right)\right) \\
x_{t} & u_{t} \\
\mu\left(y_{t}\right) & \left.=v_{t} v_{t}\left(x_{t}\right) \wedge \mu\left(u_{t}\right) \wedge \mu\left(y_{t} \mid x_{t}, u_{t}\right)\right)
\end{aligned}
$$

It should be noted that the same expressions can be obtained by applying (1.8) to (3.13) and (3.14), with the input at time $t$ assumed to be a fuzzy set characterized by $\mu\left(u_{t}\right)$.

In symbolic form, (3.28) and (3.29) can be expressed as

$$
\begin{aligned}
X^{t+1} & =F_{O O}\left(X^{t}, U^{t}\right) \\
Y^{t} & =G_{O O}\left(X^{t}, U^{t}\right)
\end{aligned}
$$

where $F_{00}$ and $G_{0 o}$ are, respectively, functions from the product space of fuzzy sets in $X$ and fuzzy sets in $U$ to the space of fuzzy sets in $X$ and fuzzy sets in $Y$. Thus, (3.30) expresses the fuzzy state at time $t+1$ as a function of the fuzzy state at time $t$ and the fuzzy input at time $t$. Similarly, (3.31) expresses the fuzzy output at time $t$ as a function of the fuzzy state at time $t$ and the fuzzy input at time $t$. Note that (3.30) is induced via (1.8) by (3.9), which expresses the fuzzy state at time $t+1$ as a function of the non-fuzzy state at time $t$ and the non-fuzzy input at time t. The same is true of (3.31) and (3.10). 
A simple numerical example will serve to illustrate the use of the formulae derived above. Specifically, let us consider a fuzzy system with binary input and output, $U=Y=\{0,1\}$, and finite state space $X=\{\alpha, \beta, \gamma\}$. Suppose that the membership functions $\mu\left(x_{t+1} \mid x_{t}, u_{t}\right)$ and $\mu\left(y_{t} \mid x_{t}, u_{t}\right)$ for this system are characterized by the following tables

\begin{tabular}{|c|c|c|c|c|c|c|}
\hline \multicolumn{1}{c|}{} & \multicolumn{3}{c|}{$u_{t}=0$} & \multicolumn{3}{c|}{$u_{t}=1$} \\
\hline$\alpha$ & $\alpha$ & $\beta$ & $\gamma$ & $\alpha$ & $\beta$ & $\gamma$ \\
\hline$\alpha$ & 1 & 0.8 & 0.6 & 0.8 & 0.5 & 1 \\
$\gamma$ & 0.7 & 0.2 & 1 & 0.2 & 1 & 0.6 \\
$\gamma$ & 0.3 & 0.3 & 0.4 & 0.9 & 0.7 & 1 \\
\hline
\end{tabular}

\begin{tabular}{|c|c|c|c|c|}
\cline { 2 - 5 } \multicolumn{1}{c|}{} & \multicolumn{2}{c|}{$u_{t}=0$} & \multicolumn{2}{c|}{$u_{t}=1$} \\
\hline $\mathrm{x}_{\mathrm{t}}$ & 0 & 1 & 0 & 1 \\
\hline$\alpha$ & 0.8 & 0.3 & 0.6 & 0.3 \\
$\beta$ & 1 & 0.1 & 0.5 & 1 \\
$\gamma$ & 0.8 & 0.7 & 0.3 & 0.2 \\
\hline
\end{tabular}

Further, assume that $\mathrm{x}^{t}$ and $\mathrm{U}^{t}$ are characterized by the membership functions tabulated below.

$$
\begin{gathered}
\mu(\alpha)=1 ; \mu(\beta)=0.8 ; \mu(\gamma)=0.4 ; \\
\mu(0)=1 ; \mu(1)=0.3 .
\end{gathered}
$$


It should be noted that, as in the case of a memoryless fuzzy system, (3.30) and (3.31) can be used to provide an approximate characterization of a non-memoryless fuzzy system. To illustrate, let us employ the convention introduced earlier, namely, using the symbol $\underset{\sim}{x}$ to denote the name of a fuzzy set or real numbers which are approximately equal to $x$. Then, viewed as relations between names of fuzzy sets, (3.30) and (3.31) may take the appearance of tables such as shown below:

\begin{tabular}{|c|c|c|c|c|c|}
\hline & $\mathrm{U}^{\mathrm{t}}$ & 1 & 2 & 3 & $\underset{\sim}{4}$ \\
\hline$x^{t+1}:$ & $\begin{array}{l}0 \\
\sim \\
\underset{\sim}{1}\end{array}$ & $\begin{array}{l}2 \\
\underset{\sim}{3}\end{array}$ & $\begin{array}{l}\underset{\sim}{2} \\
\underset{\sim}{4}\end{array}$ & $\begin{array}{l}4 \\
\tilde{1}\end{array}$ & $\begin{array}{l}3 \\
3 \\
2\end{array}$ \\
\hline
\end{tabular}

\begin{tabular}{|c|c|c|c|c|c|}
\hline \multirow{3}{*}{$\mathrm{Y}^{\mathrm{t}}:$} & $u^{t}$ & 1 & 2 & 3 & 4 \\
\hline & 0 & 0 & 1 & 1 & 0 \\
\hline & 1 & 1 & 0 & 1 & 1 \\
\hline
\end{tabular}

where for simplicity we restricted $x$ and $u$ to integral values. More generally, the entries in these tables would be names for fuzzy sets in $X$, $U$ and $Y$, and only a finite number of such names would be used as representative samples (paradigms) of the fuzzy sets in their respective spaces.

So far, we have restricted our attention to the case where a single fuzzy input $\mathrm{U}^{\mathrm{t}}$ is applied to $Q$ in state $\mathrm{x}^{\mathrm{t}}$. For this case, we found expressions for $\mathrm{X}^{\mathrm{t}+1}$ and $\mathrm{Y}^{\mathrm{t}}$ in terms of $\mathrm{X}^{t}$ and $\mathrm{U}^{\mathrm{t}}$. The same approach can readily be extended, however, to the case where the input is a sequence of non-interacting fuzzy inputs $U^{t} u^{t+1} \ldots U^{t+n}, n \geq 1$. The assumption of 
non-interaction implies that

$$
\mu\left(u_{t}, \ldots, u_{t+n}\right)=\mu\left(u_{t}\right) \wedge \mu\left(u_{t+1}\right) \wedge \ldots \wedge \mu\left(u_{t+n}\right)
$$

To illustrate, let $n=1$. Then by applying (1.8) to (3.23) and (3.24), we obtain

$$
\begin{aligned}
& \mu\left(x_{t+2}\right)=\begin{array}{ccccc}
v & v & v & v \\
x_{t+1} & x_{t} & u_{t} & u_{t+1}
\end{array}\left(\mu\left(x_{t}\right) \wedge \mu\left(x_{t+1} \mid x_{t}, u_{t}\right) \wedge\right. \\
& \left.\mu\left(x_{t+2} \mid x_{t+1}, u_{t+1}\right) \wedge \mu\left(u_{t}\right) \wedge \mu\left(u_{t+1}\right)\right) \\
& \mu\left(y_{t+1}\right)=\begin{array}{ccccc}
v & v & v & v \\
x_{t+1} & x_{t} & u_{t} & u_{t+1}
\end{array}\left(\mu\left(x_{t}\right) \wedge \mu\left(x_{t+1} \mid x_{t}, u_{t}\right) \wedge\right. \\
& \left.\mu\left(y_{t+1} \mid x_{t+1}, u_{t+1}\right) \wedge \mu\left(u_{t}\right) \wedge \mu\left(u_{t+1}\right)\right)
\end{aligned}
$$

As in the case of stochastic systems, for higher values of $n$ such relations can be expressed more compactly through the use of vector and matrix notation. For our purposes, the simple case of $n=1$ considered above suffices to illustrate the main features of the method which can be used to compute the fuzzy state and fuzzy output of a system at the end of a finite sequence of non-interacting fuzzy inputs. 
As was shown in a recent note, ${ }^{3}$ the notion of a fuzzy system bears a close relation to that of a fuzzy algorithm.

Roughly speaking, a fuzzy algorithm is an algorithm in which some of the instructions are fuzzy in nature. Examples of such instructions are: (a) Increase $x$ slightly if $y$ is slightly larger than 10;

(b) Decrease $u$ until it becomes much smaller than v; (c) Reduce speed if the road is slippery. The sources of fuzziness in these instructions are the underlined words.

More generally, we may view a fuzzy algorithm as a fuzzy system A characterized by equations of the form:

$$
\begin{array}{rlr}
\mathrm{X}^{\mathrm{t}+1} & =F\left(\mathrm{X}^{\mathrm{t}}, \mathrm{U}^{\mathrm{t}}\right) & 4.1 \\
\mathrm{U}^{\mathrm{t}} & =H\left(\mathrm{X}^{\mathrm{t}}\right) & 4.2
\end{array}
$$

where $X^{t}$ is a fuzzy state of $A$ at time $t, U^{t}$ is a fuzzy input (representing a fuzzy instruction) at time $t$, and $x^{t+1}$ is the fuzzy state at time $t+1$ resulting from the execution of the fuzzy instruction represented by $\mathrm{U}^{\mathrm{t}}$. As seen from (4.1) and (4.2), the function $F$ defines the dependence of the fuzzy state at time $t+1$ on the fuzzy state at time $t$ and the fuzzy input at time $t$, while the function $H$ describes the dependence of the fuzzy input at time $t$ on the fuzzy state at time $t$.

To illustrate (4.1) and (4.2), we shall consider a very simple example. Suppose that $X$ is a fuzzy subset of a finite set $X=$ $\left\{\alpha_{1}, \alpha_{2}, \alpha_{3}, \alpha_{4}\right\}$ and $U^{t}$ is a fuzzy subset of a finite set $U=\left\{\beta_{1}, \beta_{2}\right\}$. 
Since the membership functions of $x^{t}$ and $U^{t}$ are mappings from, respectively, $X$ and $U$ to the unit interval, these functions can be represented as points in unit hypercubes in $R^{4}$ and $R^{2}$, which we shall denote for convenience by $C^{4}$ and $C^{2}$. Thus, $F$ may be defined by a mapping from $C^{4} \times C^{2}$ to $C^{4}$ and $H$ by a mapping from $\mathrm{C}^{4}$ to $\mathrm{C}^{2}$. For example, if the membership function of $\mathrm{x}^{\mathrm{t}}$ is represented by the vector $(0.5,0.8,1,0.6)$ and that of $\mathrm{U}^{\mathrm{t}}$ by the vector $(1,0.2)$, then the membership function of $x^{t+1}$ would be defined by $F$ as a vector, say, $(0.2,1,0.8,0.4)$, while that of $\mathrm{U}^{\mathrm{t}}$ would be defined by $H$ as a vector, $\operatorname{say}(1,0.2)$.

It is clear that even in the very simple case where $X$ and $U$ are small finite sets, It is impracticable to attempt to characterize $F$ with any degree of precision as a mapping from a product of unit hypercubes to a unit hypercube. Thus, in general, it would be necessary to resort to an approximate definition of $F$ and $H$ through the process of exemplification, as was done in the case of the relation between $Y^{t}$ and $U^{t}$ in Chapter 3 (Eq. (3.8) et seq.). This amounts to selecting a finite number of sample fuzzy sets in $X$ and $U$, and tabulating finite approximations to $F$ and $H$ as mappings from and to the names of these fuzzy sets. In this light, an instruction such as "Reduce speed if the road is slippery" may be viewed as an ordered pair in $\mathrm{H}$ involving the names of fuzzy sets: "Reduce speed" and "Road is slippery."

Consider now the following situation. One is given an instruction of the form: "If $x$ is much larger than 1 make $y$ equal to 2 . Otherwise make y equal to 1." Furthermore, the membership function of the class of numbers which are much larger than 1 is specified to be

$$
\begin{array}{rlrl}
\mu_{E}(x) & =0 & & \text { for } x<1 \\
& =\left(1+(x-1)^{-2}\right)^{-1} & \text { for } x \geq 1
\end{array}
$$

where $E$ denotes the class in question and $\mu_{E}$ is its membership function. 
Now suppose that $x=3$. How should the above instruction be executed? Note that $\mu_{E}(3)=0.8$.

The answer to this question is that the given instruction does not cover this contingency or, for that matter, any situation in which $x$ is a number such that $\mu_{E}(x)>0$. Specifically, the instruction in question tells us only that if the input is a fuzzy set characterized by the membership function (4.3), then $y=2$; and if the input is characterized by the membership function $1-\mu_{E}(x)$, then $y=1$. Now when $x$ is specified to be equal to 3 , the input may be regarded as a fuzzy set whose membership function is equal to 1 for $x=3$ and vanishes elsewhere. This fuzzy set is not in the domain of the instruction - viewing instruction as a function defined on a collection of fuzzy sets.

In some cases, it may be permissible to extend the domain of definition of a fuzzy instruction by an appropriate interpretation of its intent. For example, in the case considered above it may be reasonable to assume that $y=2$ not just for the fuzzy set of numbers which are much larger than 1, but also for all fuzzy subsets of this set whose maximal grade of membership exceeds or is equal to a prescribed threshold; or, it may be reasonable to assume that $y=2$ for all $x$ whose grade of membership in $E$ is greater than or equal to a threshold $\alpha$. Alternatively, the domain of the instruction can be extended by employing randomized execution, that is, by choosing $y=2$ and $y=1$ for a given $x$ with probabilities $\mu_{E}(x)$ and $1-\mu_{E}(x)$, respectively. These and other ways of extending the domain of fuzzy instructions make the specification of $F$ and $H$ a problem which, though nontrivial, is well within the range of computational feasibility in many cases of practical interest.

Actually, crude forms of fuzzy algorithms are employed quite extensively in everyday practice. A cooking recipe is an example of an algorithm of this type. So is the set of instructions for parking a car or repairing a $\mathrm{TV}$ set. The effectiveness of such algorithms depends in 
large measure on the existence of a fuzzy feedback which makes it possible to observe the output and apply a corrective input. Indeed, this is implicit in equation (4.3), except that in practice the $\mathrm{H}$ function is itself quite 1ll-defined.

The foregoing discussion of the notion of a fuzzy algorithm was intended primarily to point to a close connection between this notion and that of a fuzzy system. It may well turn out, however, that many of the complex problems - such as machine translation of languages - which so far have eluded all attempts to solve them by conventional techniques cannot be properly formulated, much less solved, without the use, in one form or another, of a broader conceptual framework in which the notion of a fuzzy algorithm plays a basic role. 


\section{CHAPTER V}

\section{THE CONCEPT OF AGGREGATE}

As was pointed out in Chapter 2, the state of a system may be viewed as a name for an aggregate of input-output pairs. In what follows, we shall summarize some of the principal notions relating to the concept of an aggregate, but will leave open the question of how these notions can be extended to fuzzy systems.

As in Chapter 2, let $\mathrm{u}$ and $\mathrm{y}$ denote a pair of sequences $\mathrm{u}=\mathrm{u}_{0} \mathrm{u}_{1} \ldots \mathrm{u}_{\mathrm{t}}$ and $\mathrm{y}=\mathrm{y}_{0} \mathrm{y}_{1} \ldots \mathrm{y}_{\mathrm{t}}$ of length $t+1$, where, for simplicity, $t$ is assumed to range over non-negative integers. If $u=u_{0} u_{1} \ldots u_{\tau}$ and $v=v_{T+1} \cdots v_{t}$, then the concatenation of $u$ and $v$ is denoted by uv and is defined by $u v=u_{0} u_{1} \ldots u_{\tau} v_{\tau+1} \ldots v_{t}$.

Definition of a system. A system (discrete-time system) $Q$ is defined as a collection of ordered pairs of time-functions $(u, y)$ satisfying the condition of closure under segmentation or CUS for short. Thus,

$$
Q=\{(\mathrm{u}, \mathrm{y})\}, \mathrm{u} \in \mathrm{U}^{*}, \mathrm{y} \in \mathrm{x}^{*}
$$

where $u$ and $y$ are, respectively, the input and output of $Q$, and (u,y) is an input-output pair belonging to $A$. The expression for the cus condition is:

If $\mathrm{u}=\mathrm{v} \mathrm{v}^{\prime}$ and $\mathrm{y}=\mathrm{ww} \mathrm{w}^{\prime}$ (that is, $\mathrm{u}$ is a concatenation of time functions $v$ and $v^{\prime}$ and $y$ is a concatenation of $w^{\prime}$ and $\left.w^{\prime}\right)$ and $(u, y) \in Q$, then $(v, w) \in Q$ and $\left(v^{\prime}, w^{\prime}\right) \in Q$. In effect, this condition requires that every segment of an input-output pair of $Q$ be an input-output pair of $A$.

Comment. When we define a system as a collection of input-output pairs, we are in effect identifying a physical system or a mathematical model of 
it with the totality of observations which can be made of its input and output time-functions. Furthermore, we tacitly assume that we have as many copies of the system as there are different initial states, and that each $\mathrm{u}$ is applied to all these copies, so that to each $\mathrm{u}$ correspond as many $y^{\prime} s$ as there are copies of the system.

To characterize $Q$ as a collection of input-output pairs it is usually more expedient to employ an algorithm for generating inputoutput pairs belonging to $A$ than to list them. From this point of view, a differential or difference equation relating the output of a system to its input may be viewed as a compact way of specifying the collection of input-output pairs which defines $A$. An algorithm or an equation which serves this purpose is called an input-output relation.

Definition of an aggregate. Let $Q\left(t_{0}\right)$ denote a subset of $Q$ comprising those input-output pairs which start at time $t_{0}$. Now suppose we group together those input-output palrs in $Q\left(t_{0}\right)$ which have some property in common and call such groups bundles of input-output paris. As we shall see presently, the aggregates of $Q$ are bundles of input-output pairs with certain special properties which are defined in such a way as to make a state of $Q$ merely a name or a label for an aggregate of $Q$.

It is convenient to state the properties in question as a set of four conditions defining aggregates of $Q$. These conditions are as follows :

1. Covering condition. Let a generic bundle of input-output pairs in $Q\left(t_{0}\right)$ be denoted by $a_{\alpha_{0}}\left(t_{0}\right)$, with $\alpha_{0}$ serving as an identifying tag for a bundle. A collection of such bundles will be denoted by $\left\{Q_{\alpha_{0}}\left(t_{0}\right)\right\}$, $\alpha_{0} \in \Sigma_{t_{0}}$, where $\Sigma_{t_{0}}$ is the range of values which can be assumed by $\alpha_{0}$ at $t_{0}$. Anticipating that $\alpha_{0}$ will play the role of a state of $A_{,} \Sigma_{t_{0}}$ will be referred to as the state space of $Q$ at time $t_{0}$. Note that $t_{0}$ is a variable ranging over the integers $0,1,2, \ldots$. 
The covering condition requires that the collection $\left\{a_{\alpha_{0}}\left(t_{0}\right)\right\}$, $\alpha_{0} \in \Sigma_{t_{0}}$, be a covering for $Q_{\left(t_{0}\right)}$, that is,

$$
{\underset{\alpha}{\alpha}}_{0} a_{\alpha_{0}}\left(t_{0}\right)=a_{\left(t_{0}\right)}, \text { for all } t_{0} \text { in }\{0,1, \ldots\} .4 .4
$$

In effect, this condition requires that every input-output pair in $a_{\left(t_{0}\right)}$ be included in some bundle in the collection $\left\{a_{\alpha_{0}}\left(t_{0}\right)\right\}, \alpha_{0} \in \Sigma_{t_{0}}$.

2. Uniqueness condition. The uniqueness condition is expressed by

$$
(u, y) \in a_{\alpha_{0}}\left(t_{0}\right) \quad \text { and } \quad\left(u, y^{\prime}\right) \in a_{\alpha_{0}}\left(t_{0}\right) \rightarrow y=y^{\prime} \cdot \quad 4.5
$$

In other words, to each input $u$ in the domain of the relation $a_{\alpha_{0}}\left(t_{0}\right)$ corresponds a unique output $y$. (Note that the sequences $u$ and $y$ are assumed to be of the same length.)

3. Prefix condition. Consider an input-output pair (uu', yy') in $a_{\alpha_{0}}\left(t_{0}\right)$ which is a concatenation of the input-output pairs $(u, y)$ and $\left(u^{\prime}, y^{\prime}\right)$. The expression for the condition is

$$
\left(u^{\prime}, y^{\prime}\right) \in a_{\alpha_{0}}\left(t_{0}\right) \rightarrow(u, y) \in a_{\alpha_{0}}\left(t_{0}\right)
$$

Thus, this condition requires that any prefix (i.e., $(u, y)$ ) of an inputoutput pair in $a_{\alpha_{0}}\left(t_{0}\right)$ also be an input-output pair in $a_{\alpha_{0}}\left(t_{0}\right)$.

4. Continuation condition. As in the preceding condition, let (uu',yy') be an input-output pair in $a_{\alpha_{o}}\left(t_{0}\right)$, with ( $u^{\prime}, y^{\prime}$ ) starting at, say, $t_{1}$. The continuation condition may be expressed as

$$
\left\{\left(u^{\prime}, y^{\prime}\right) \mid\left(u u^{\prime}, y^{\prime}\right) \in a_{\alpha_{0}}\left(t_{0}\right)\right\}=a_{\alpha_{1}}\left(t_{1}\right)
$$



If we could find an answer to this basic question, we might, perhaps, be able to develop effective techniques for the approximate analysis of complex systems for which state equations cannot be postulated at the outset. We state this question as an open problem because its solution can be perceived only dimly at this rudimentary stage of the development of the theory of fuzzy systems. 


\section{BIBL IOGRAPHY}

(1) Zadeh, L. A., "Fuzzy Sets," Information and Control, Vol. 8, pp. 338-353, June 1965.

(2) Zadeh, L. A., "Fuzzy Sets and Systems," Proc. of the Symposium on System Theory, Polytechnic Institute of Brooklyn, New York, N.Y., pp. 29-39, April 1965.

(3) Zadeh, L. A., "Fuzzy Algorithms," Information and Control, Vol. 12, pp. 99-102, February 1968.

(4) Santos, E. S. and Wee, W. G., "General Formulation of Sequential Machines," Information and Control, Vol. 12, pp. 5-10, 1968.

(5) Santos, E. S., "Maximin Automata," Information and Control, Vol. 13, pp. 363-377, October 1968 .

(6) Goguen, J., "L-Fuzzy Sets," Jour, Math. Anal. and App1., Vo1. 18, pp. 145-174, April 1967.

(7) Zadeh, L. A., "Shadows of Fuzzy Sets," Problems in Transmission of Information (in Russian), Vo1. 2, pp. 37-44, March 1966.

(8) Zadeh, L. A. and Desoer, C. A., "Linear System Theory - The State Space Approach," McGraw-Hil1 Book Co., New York, N.Y., 1963.

(9) Zadeh, L. A., "The Concept of State in System Theory," in Network and Switching Theory," ed. G. Biorci, Academic Press, New York, N.Y., 1968.

(10) Zadeh, L. A., "The Concepts of System, Aggregate and State in System Theory," in System Theory, eds. L. A. Zadeh and E. Polak, McGraw-H111 Book Co., New York, N.Y., 1969. 
(11) Carlyle, J. W., "Reduced Forms for Stochastic Sequential Machines," Jour. Math. Anal. and Appl., Vol. 7, pp. 167-175, 1963. 